\title{
"Wi-Rover": A Wi-Fi Controlled Remotely Operated Video Enhanced Receiver
}

\author{
Sudarshan B T \\ Mtech in Software \\ Engineering, RVCE \\ Bangalore, India
}

\author{
Namratha M \\ Mtech in Software \\ Engineering, Department of \\ Information Science, PESIT \\ Bangalore, India
}

\author{
Pradeep \\ Mtech in Software \\ Engineering, Department of \\ Information Science, PESIT \\ Bangalore, India
}

\begin{abstract}
The aim of the system is to demonstrate the use of wireless digital communication over the internet to control and communicate with a vehicular system. Design a rover which has the capability to navigate based on the commands issued to it remotely by an operator. Rover should provide a communication interface to send and receive data to the operator. This communication must be in real time with as minimal a delay as possible. Rover should be aware of its status, which it should communicate to the operator. The authenticity of the operator should be verified for communication establishment. The communication between the client and the rover should be established based on the connection parameters specified by the operator. Secure and Real time communication of control data and rover status data between the rover and the client to ensure real time operations.
\end{abstract}

\section{General Terms}

Wireless digital communication, Real time communication, Wi-fi, Autonomous vehicle

\section{Keywords}

Rover, Linksys, OpenWRT, Arduino microcontroller, Proximity sensors, Zavio F210A

\section{INTRODUCTION}

A rover is an exploration vehicle designed to move across the surface of a planet or other dangerous surface. Some rovers have been designed to transport members of a human crew; others have been partially or fully autonomous robots. There are also several robots that are similar to these rovers and are used for bomb-disposal and other exploratory activities. They are generally very expensive to build and are for a single purpose only. They use analog networks and are are not scalable. Once built, they are difficult to repurpose.

Also autonomous transport has been a one of the primary goals of technology. Several attempts have been made to create a transport system that is completely autonomous. This has been difficult to achieve due to the lack of reliable and robust communication between the central controller that controls all these vehicles. Also some form of co-ordination between the remotely controlled vehicles is also needed. There is also a need for complex algorithms that route the vehicles safely and quickly. All these can be achieved by modern technologies. Global Positioning System can be used to plot the location of the devices and this information can be sent to a central controller through wireless networks. This controller then based on all the information that it has regarding the vehicles decides the path that should be taken. Since all this information is in the digital format it is easily extensible and scalable. But some form of autonomous control is also needed in the vehicle. Say if it detects that it is too close to some structure behind or ahead of it then it should slow down or stop completely. Also if the vehicle is running out of power the routing should be changed so that the vehicle and go to the closest refuelling station on the way and recharge. Thus there is a need for a two-way communication between the vehicle and the controller. Modern communication networks and large supercomputer can easily achieve all of this without difficulty. Thousands of such vehicles can be controlled in this fashion.

There have been several advances in this field of late. The VANET(Vehicular Adhoc Network) consortium is developing a network that can be used in developing a adhoc network among cars that move on the road so that there is efficient usage of the roadways( ex: traffic jams may be avoided, if there is an accident even on a remote location. BMW of Germany along with BOSCH has developed a car that can be controlled through GPS satellites. Once a human takes a lap of the circuit then the car's computer along with the GPS can then do an exact replication of the stored lap. It has also been proven that the accuracy and safety due to these technologies are more than a human controlled one. If these technologies can be integrated then a completely autonomous transportation system can be developed.

\section{LITERATURE SURVEY}

Large scale research is underway to improve remote rover technology especially for applications in urban environments. These applications maybe as varied as aiding various military forces to creating driverless cars[7] that can be controlled externally.

ROVER, which stands for Remotely Operated Video Enhanced Receiver, is a system which allows ground forces, such as Forward Air Controllers (FAC), to see what an aircraft or unmanned aerial vehicle (UAV) is seeing in real time by receiving images acquired by the aircraft's sensors on a laptop on the ground. There's little time delay and usage of ROVER greatly improves the FAC on the ground reconnaissance and target identification which are essential to close air support.

For years a lot of investment has been made in terms of time and money to develop rovers that can easily and efficiently deliver. Three US Government funded military efforts known as Demo I (US Army), Demo II (DARPA), and Demo III (US Army), are currently underway. Demo III (2001) demonstrated the ability of unmanned ground vehicles to navigate miles of difficult off-road terrain, avoiding obstacles such as rocks and trees. James Albus at NIST provided the Real-Time Control System which is a hierarchical control system. Not only were individual vehicles controlled (e.g. throttle, steering, and brake), but groups of vehicles had their 
movements automatically coordinated in response to high level goals.

As of 2007, a three-year project is underway to allow robot[10] controlled vehicles, including buses and trucks, to use a special lane along 20 Interstate 805 . The intention is to allow the vehicles to travel at shorter following distances and thereby allow more vehicles to use the lanes. The vehicles will still have drivers since they need to enter and exit the special lanes. The system is being designed by Swoop Technology, based in San Diego[9] county.

A driverless car is an autonomous vehicle that can drive itself from one point to another without assistance from a driver; in other words, with an autopilot system. Some believe that autonomous vehicles have the potential to transform the transportation industry while virtually eliminating accidents, and cleaning up the environment. According to urban designer and futurist Michael E. Arth, driverless electric vehicles - in conjunction with the increased use of virtual reality for work, travel, and pleasure - could reduce the world's 800,000,000 vehicles to a fraction of that number within a few decades. Arth claims that this would be possible if almost all private cars requiring drivers, which are not in use and parked $90 \%$ of the time, would be traded for public self-driving taxis that would be in near constant use. This would also allow for getting the appropriate vehicle for the particular need - a bus could come for a group of people, a limousine could come for a special night out, and a Segway could come for a short trip down the street for one person. Children could be chauffeured in supervised safety, DUIs would no longer exist, and 41,000 lives could be saved each year in the U.S. alone.

Driverless passenger car programs include the 800 million EC EUREKA Prometheus Project on autonomous vehicles (19871995), the 2getthere passenger vehicles (using the FROGnavigation technology) from the Netherlands, the ARGO research project from Italy, and the DARPA Grand Challenge from the USA.

In order to drive a car a system would need to:

1. Understand its immediate environment (Sensors)

2. Know where it is and where it wants to go (Navigation)

3. Find its way in the traffic (Motion planning)

4. Operate the mechanics of the vehicle (Actuation)

Usually $21 / 2$ of these problems are already solved: Navigation and Actuation completely, and Sensors partially, but improving fast. The main unsolved part is the motion planning.

NASA's Mars Exploration Rover Mission (MER), is an ongoing robotic space mission involving two rovers, Spirit and Opportunity, exploring the planet Mars. It began in 2003 with the sending of the two rovers MER-A Spirit and MER-B Opportunity to explore the Martian surface and geology.

The mission's scientific objective was to search for and characterize a wide range of rocks and soils that hold clues to past water activity on Mars. The mission was part of NASA's Mars Exploration Program which includes three previous successful landers: the two Viking program landers in 1976 and Mars Pathfinder probe in 1997.

The rovers are six-wheeled, solar-powered robots. Each rover has six wheels mounted on a rocker-bogie suspension system that ensures wheels remain on the ground while driving over rough terrain. The rover needs about 100 watts to drive. Its power system includes two rechargeable lithium ion batteries weighing $7.15 \mathrm{~kg}$ (16 pounds) each, that provide energy when the sun is not shining, especially at night. The rover has a lowgain and a high-gain antenna. The low-gain antenna is omnidirectional, and transmits data at a low rate to Deep Space Network (DSN) antennas on Earth. The high-gain antenna is directional and steerable, and can transmit data to Earth at a higher rate.

The rovers also use the low-gain antennas to communicate with spacecraft orbiting Mars, the Mars Odyssey and (before its failure) the Mars Global Surveyor. The orbiters relay data from and to Earth; most data to Earth is relayed through Odyssey. The benefits of using the orbiters are that they are closer to the rovers than the antennas on Earth, and have view of Earth for much longer than the rovers. The orbiters communicate with the rovers using UHF antennas, which have shorter range than the low and high-gain antennas. One UHF antenna is on the rover and one is on a petal of the lander to aid in gaining information during the critical landing event.

The rovers have a total of 9 cameras, which produce 1024 pixel by 1024-pixel images at 12 bits per pixel, but most navigation camera images and image thumbnails are truncated to 8 bits per pixel to conserve memory and transmission time.

All images are then compressed using ICER before being stored and sent to Earth. Navigation, thumbnail, and many other image types are compressed to approximately 0.8 to 1.1 bits/pixel. Lower bit rates (less than $0.5 \mathrm{bit} / \mathrm{pixel}$ ) are used for certain wavelengths of multi-color panoramic images.

A team of researchers at the Center for Automotive Research at Stanford (CARS)[8] has filled the trunk of an Audi TTS with computers and GPS receivers, transforming it into a vehicle that drives itself. The car will attempt Pikes Peak without a driver at race speeds, something that's never been done.

The Stanford Racing Team won its first autonomous race in 2005 with Stanley, a car developed for the Grand Challenge held in the Mojave Desert by the Defense Advanced Research Projects Agency (DARPA). Their second car, Junior, built in collaboration with Volkswagen, took second place in DARPA'S 2007 Urban Challenge.

The Audi that will attempt Pikes Peak is named Shelley after Michèle Mouton, the first female driver to win the uphill climb. Unlike Stanley and Junior, who sense the road with radars and cameras, Shelley will follow a GPS trail from start to finish. The trick will be to stay on the road at race speeds while sliding around the corners.

Shelley knows exactly where she is on the road by using a differential GPS. Unlike a standard GPS system, hers corrects for interference in the atmosphere, showing the car's position on the Earth with an accuracy of about 2 centimeters. Shelley measures her speed and acceleration with wheel-speed sensors and an accelerometer, and gets her bearings from gyroscopes, which control equilibrium and direction.

CARS is funded by Volkswagen, Bosch, Honda, Toyota and Nissan. The work done so far varies significantly in its ambition and its demands in terms of modification of the infrastructure. Broadly, there are three approaches:

- Fully autonomous vehicles 
- Various enhancements to the infrastructure (either an entire area, or specific lanes) to create a selfdriving closed system.

- "assistance" systems that incrementally remove requirements from the human driver (e.g. improvements to cruise control)

Fully autonomous driving requires a car to drive itself to a pre-set target using unmodified infrastructure. The final goal of safe door-to-door transportation in arbitrary environments is not yet reached though.

\section{DESIGN CONSIDERATIONS}

Rover technology has always been the forte of wireless communication, several varied techniques having already been used to wirelessly control a rover, which would be located from a few meters to several hundred million kilometers away from the controller[6]. An interesting offshoot is the attempt to control the rover, not using a dedicated signal link unique to the rover-controller, but instead, a generic mechanism which connects the rover to its controller much the same way as it would connect a wireless device to another. The technology that immediately became relevant in this context was the Wireless Fidelity or Wi-Fi.

Wi-Fi, which is used as a synonym for the IEEE 802.11 Wireless communication technology standard, is widely used at present to interconnect virtually all mobile devices, desktops and other peripheral devices. Wi-Fi coverage ranges from a square meters to several square miles depending upon the access-point topology within the area in question, which makes it ideal to use as the medium between the rover and its controller. The motivation of this project therefore, is the exciting application of $\mathrm{Wi}-\mathrm{Fi}$, mentioned above, which would revolutionize how a rover can be controlled, i.e. possibly with just a laptop or a handheld mobile device, and its positive implications to the development process of the rover itself.

The overall approach is to develop a prototype technology demonstrator. Each module of the prototype is developed individually; coding of the microcontroller and the clientserver applications, some electronic circuitry involves simple simulations before they are fabricated The technology demonstrator could be generalized to any type of rover, from a small toy car driven by $3 \mathrm{~V}$ motors, to a heavily armored unmanned battle tank, to even small terrain hugging unmanned aerial vehicles. Considering that fact for its facevalue, it can be conclude that the technology could be used widely to deploy assets in an area seamlessly, with little or no prerequisites, thus making it a valuable technology to delve into, more deeply.

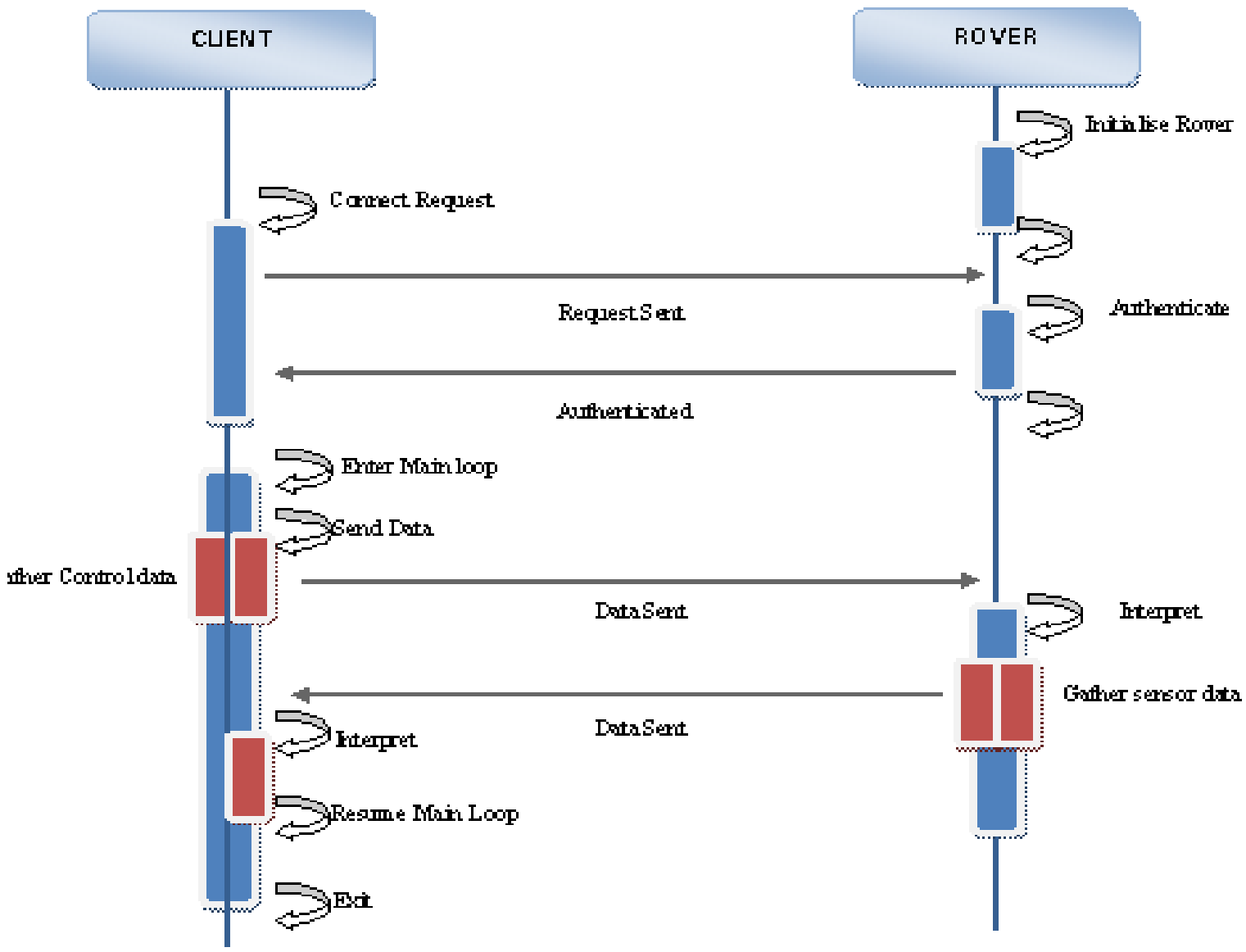

Figure 1: Interaction sequence diagram for the interaction between the clients and the rover 


\subsection{Router}

The Interface between the rover and the outside world, the router is the most important component encapsulated in the rover. The router has the capability of tethering itself to a $\mathrm{WiFi}$ access point as a client. The router must have the ability to provide multiple nodes, each sharing its Ethernet connection.

The interpretation of the data received from the client needs to be done in the router since the internal entities of the rover do not interpret the language of the clients. Thus a minimal processing capacity is required on the router. Also the firmware of the router needs to be highly configurable so that it can be tweaked to suit the needs, as and when required. The preference is for a router that uses certain Free and Open Source Software (FOSS) in its implementation. This facilitates the use of FOSS firmware for the router.

The Linksys[2] WRT54GL is a wireless router developed by the CISCO Systems which uses FOSS components thus mandating that the implementation of the router itself is open sourced. There are many simple Linux firmware distros available which can be flashed onto the Linksys and used to configure the router. The OpenWRT-White Russian[3] is one such Linux based firmware which provides various options to configure the router as desired. OpenWRT[4] also provides the required capability of interpreting the data received from the client since it completely supports POSIX compliant C APIs which can be used to program a server to run on the Linksys. Also it is quite simple to add an RS-232 serial port to the Linksys, which is most essential for communication between the router and the microcontroller.

\subsection{Microcontroller}

The Microcontroller is the next level of abstraction in the rover. The router interprets the client data and sends the interpreted data to the microcontroller. The microcontroller converts the digital data into the analog signals which activate the actuators on the rover. It generally uses assembly language to accomplish this. An additional functionality required on the microcontroller is to control the speed of the rover. This also needs to be implemented on the microcontroller.

The microcontroller needs a set of Analog-to-Digital converters to read the sensor inputs and interpret them. The sensors provide analog values at a certain voltage level, indicating their corresponding parameters and the microcontroller reads these sensor values from the corresponding input pins as digital values and sets the status accordingly.

The Arduino[1] is single-board microcontroller and a software suite for programming it. The hardware consists of a simple open hardware design for the controller with an Atmel AVR processor and on-board I/O support. The software consists of a standard programming language and the boot loader that runs on the board. Arduinos are programmed using the Wiring language, which is essentially $\mathrm{C}++$ with a few simplifications.

An Arduino board consists of an 8bit Atmel AVR microcontroller with complementary components to facilitate programming and incorporation into other circuits. An important aspect of the Arduino is the standard way that connectors are exposed allowing the CPU board to be connected to a variety of interchangeable add-on modules (known as shields). Official Arduinos have used the megaAVR series of chips, specifically the ATmega8, ATmega168,ATmega328 and ATmega1280.

An Arduino's microcontroller is also pre-programmed with a bootloader that simplifies uploading of programs to the onchip flash memory, compared with other devices that typically need an external chip programmer.

The Arduino IDE is a cross-platform application written in Java which is derived from the IDE made for the Processing programming language and the Wiring project. It is designed to introduce programming to artists and other newcomers unfamiliar with software development. It includes a code editor with features such as syntax highlighting, brace matching, and automatic indentation, and is also capable of compiling and uploading programs to the board with a single click. There is typically no need to edit Makefiles or run programs on the command line.

The Arduino IDE comes with a $\mathrm{C} / \mathrm{C}++$ library called "Wiring" (from the project of the same name), which makes many common input/output operations much easier. Arduino programs are written in $\mathrm{C} / \mathrm{C}++$, although users only need to define two functions in order to make a runnable program:

- $\quad \operatorname{setup}()$ - a function run once at the start of a program which can be used for initializing the output pins and also to set the baud rate of the Serial port.

- $\operatorname{loop}()-$ a function called repeatedly until the board is powered off.

The Arduino is a reliable FOSS which can be used to do away with cumbersome assembly level programming and at the same time making it easy to code and debug the microcontroller. The interaction between the microcontroller and the actuator circuit is simple and it does not need to be represented as a sequence diagram. Similarly the gathering of sensor data by the microcontroller is also a simple process of reading the analog value on the sensor's output pin, this does not need to be represented by a sequence diagram either.

\subsection{IP camera}

The camera is the eye of the rover ofcourse. The forward looking camera on a rover is equipped to help the operators to control the rover remotely. The video stream from the camera is continually streamed to the operator's interface in real-time to help in navigation.

The main requirement in the camera is its ability to connect to the router through the ethernet. The use of ethernet to retrieve the live video stream simplifies the problem of routing the video stream to the client, which would have been difficult without the ethernet access (through serial or parallel port communication). The router is directly connected to the camera through an Ethernet Port. The communication between the camera and router is very simple and does not need to be represented using a sequence diagram. The Zavio F210A is an ultra compact IP network camera featuring a high sensitivity progressive scan CMOS sensor. This IP camera achieves image quality and low-light performance previously only seen in CCD cameras. This IP network camera possesses all the powerful features of the larger professional network cameras though it is one of the world's most compact IP cameras. The F210A provides easy ethernet access and various options for configuring the camera for our needs. 


\subsection{Battery system}

The rover consists of various components each of which draw diffenrt amounts of power from the batteries. The three main components on the rover are the router, the microcontroller and the camera. As can be seen, each component draws different amounts of power. Thus it is required to design a consolidated and robust battery system which can be used to power the components instead of using individual batteries for each component which would increase the dead weight of the rover.

A single battery pack is thus designed which drives a voltage regulator which supplies regulated DC voltage levels to each of the individual components. The design of the battery voltage regulator circuitry is as shown in the following page. The battery pack consists of three batteries each rated at $7 \mathrm{~V}$ 2A. Two of these batteries are connected in series with the combined rating of $14 \mathrm{~V}-2 \mathrm{~A}$ sufficient to power router and the microcontroller. The other battery independently powers the camera.

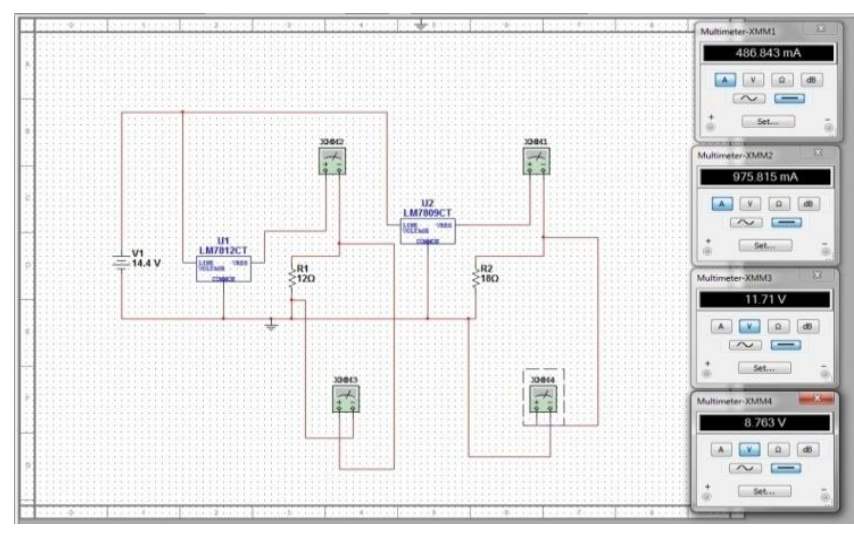

Figure 2: MultiSim simulator

\subsection{Battery Level Meter}

The discharge level of the battery pack is essential for smooth working of the rover. Thus it is a necessity to monitor the discharge level of the battery pack, so that it can be recharged timely and malfunction within the rover due to low power supply issues can be avoided.

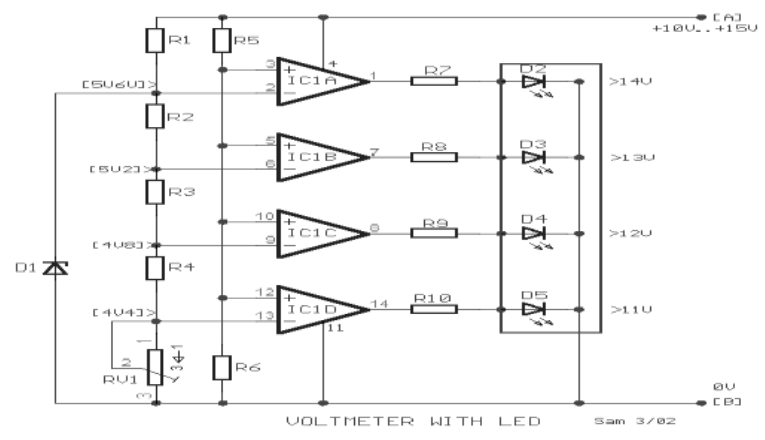

Figure 3: Simplest battery level indicator circuits

The battery pack, when fully charged, has a voltage level slightly above the rated voltage and when fully discharged it has a voltage level slightly below the rated voltage. The above circuit uses this characteristic to sense how the battery discharges. The circuit provides a visual indicator using LEDs and also output lines which can be read from.

The comparators are used to compare the voltage level of the battery pack to a set of reference voltages. Based on the voltage levels the LEDs glow and the corresponding lines are HIGH. The leads from the LED are also used by the microcontroller to do an analog-read on the lines to extract the present voltage level (all lines HIGH indicating a fully charged battery), the topmost LED in the circuit indicating the Most Significant Bit. As the battery discharges, the LEDs switch off one after the other starting from the MSB.

\subsection{Proximity sensor}

The rover, with only its forward looking camera, is blind to its baffles or its rear end. This makes it difficult to avoid obstacles while reversing the rover. Therefore it is prudent to either include a camera with a panoramic view or to include proximity sensors on the rover which sense for the proximity of the rover to obstacles, thus helping in taking counter measures.

The simplest among the sensors used for obstacle avoidance are the InfraRed LED-Phototransistor couple. These sensors consist of an IR LED and a phototransistor mounted usually on a Thin Small Outline Package.

The IR LED emits IR waves continuously which are reflected from obstacles onto the phototransistor. When the reflected IR waves are intense enough, which means to say there is an obstacle too close to the rover for comfort, the out pin of the sensor sets to HIGH. The range of the sensor depends on the quality of the product itself ofcourse. Thus based on a sensitivity required, the sensor is chosen.

\subsection{Clients}

The components in one major high level entity have been discussed in the Front-end Client design. The client handles all the controls for the rover and also encapsulates various widgets necessary to indicate the status of the rover itself.

The characteristics of the client application depend upon the system which hosts the client itself. The use of Wi-Fi communication to implement wireless communication between the client and the server results in two major cases:

1. The system running the client has internet connectivity and is present on the same sub network as the rover.

2. The system running the client has internet connectivity and is not present on the same sub network as the rover.

In the former case, the communication with the rover becomes simple since the local IP address of the router is known within the subnet. Thus the development of the client to operate in the same subnet as the rover can be treated as the first iteration in the development of a comprehensive client application. The Desktop client implements the first situation, while the Web client is designed to address both the situation with equal ease. The Web client is discussed later. 


\subsection{Desktop client}

The first iteration of the client development is implemented as a Desktop Client which is deployed on a machine as a desktop application. The process of developing the front end of the client is simplified with the use of the various IDEs available.

The interface to a rover should include the following basic components,

1. Widgets to enter connection parameters.

2. Widgets to control the rover motion and its speed settings.

3. Widget to render the live video feed from the rover.

4. Widgets to indicate the status of the rover.

These are the basic components that are a fixture in the clients and are absolutely essential for the rover's efficient operation.

The main operations to be addressed during the design of the client are the data communication between the client and the router and the event handling in the front end, along with the updation of the rover status widgets.

The main objective while designing the interface is to separate the components that handle the events and the components that implement the data communication. This is because of the following reasons,

1. If the two features are combined, then the communication has to be synchronous since every event needs to be intimated to the rover.

2. The client will be blocked from handling events until the previous event is completely handled. This results in real time delay in handling the events efficiently.

3. Also any delay anywhere in the sequence of execution may result in race conditions where multiple events go unhandled and the final state of the router becomes unpredictable.

When the two components mentioned above are made independent of each other the events can be handled almost instantaneously and the status word indicating the control is sent to the rover periodically so that, all changes made to the status word during the interval are consolidated into a single final state which is then sent to the rover. All the above mentioned problems are overcome by using this paradigm.

The client provides widgets to enter the connection parameters such as the IP address, port number and the access password. It also provides a widget to select the speed of the rover. It handles any key event and if they are corresponding to the control keys then it updates the status word.

The client receives the live video feed from the rover and needs to render it. The client also receives the status word indicating the status of the rover. The status of the rover considered here are the Reverse Disable Status and the Battery level indication. These parameters have their own widgets to indicate their status. Communication is handled using sockets which connects and binds the client and server.
C\#.NET[5] is ideal for the development of the desktop client. The Microsoft Visual Studio IDE provides extensive options to create and deploy the client in an efficient manner. The advantages of using the $\mathrm{C} \#$ platform are,

1. .NET platform is available which provides crossplatform capabilities.

2. The tools provided by $\mathrm{C \#}$ are excellent for the development of the front end.

3. Helps in the rapid deployment of a reliable cross platform interface.

\subsection{Web client}

The Desktop interface client formerly introduced and described is a tool suitable to connect to the remote server if the server resides in the same sub-network as the system running the client itself. But if the server and the client are present on two different networks, it requires more elaborate addressing to gain access to the server.

This necessitates the use of an interface which can handle communication over the internet in a simple and elegant manner. The web interface is designed to connect to the server program which runs on the router. This leads to the design of an interface as an HTML document which handles the frontend functionalities of the interface using embedded JavaScript, while the back-end is handled by a simple program, written in PHP, which acts as a bridge between the server and the client. The Web client encapsulates the same widgets as described in the Desktop client. The interface between the front and the back-ends is implemented using AJAX. Together these two components handle the simple functionalities of the interface.

The Web client is more complex when compared to the Desktop client.. The JavaScript processing in the client side consists of two main logical components similar to the desktop client. One is the component which handles the data communication between the client and the server side. This is accomplished using the Timer function. The other main logical component is the section of code which updates the control status word according to the key events. This part of the code is independent of the Timer function. This is advantageous since the status word need not be sent everytime a key event occurs. This not only makes the implementation cumbersome but also unnecessarily increases the bandwidth required.

The communication between the JavaScript and the PHP must be asynchronous to separate the event handling and data transfer functions. AJAX-XMLHTTP request-response is ideal for implementing such asynchronous communication. The status word is updated for every key event and the consolidated status word at the instance of the timeout is sent to the rover. The status of the Rover is received from the PHP as a XMLHTTP response text and its value can be used to update the status of the front end to reflect the status of the rover. The PHP uses sockets to connect to the rover directly. 


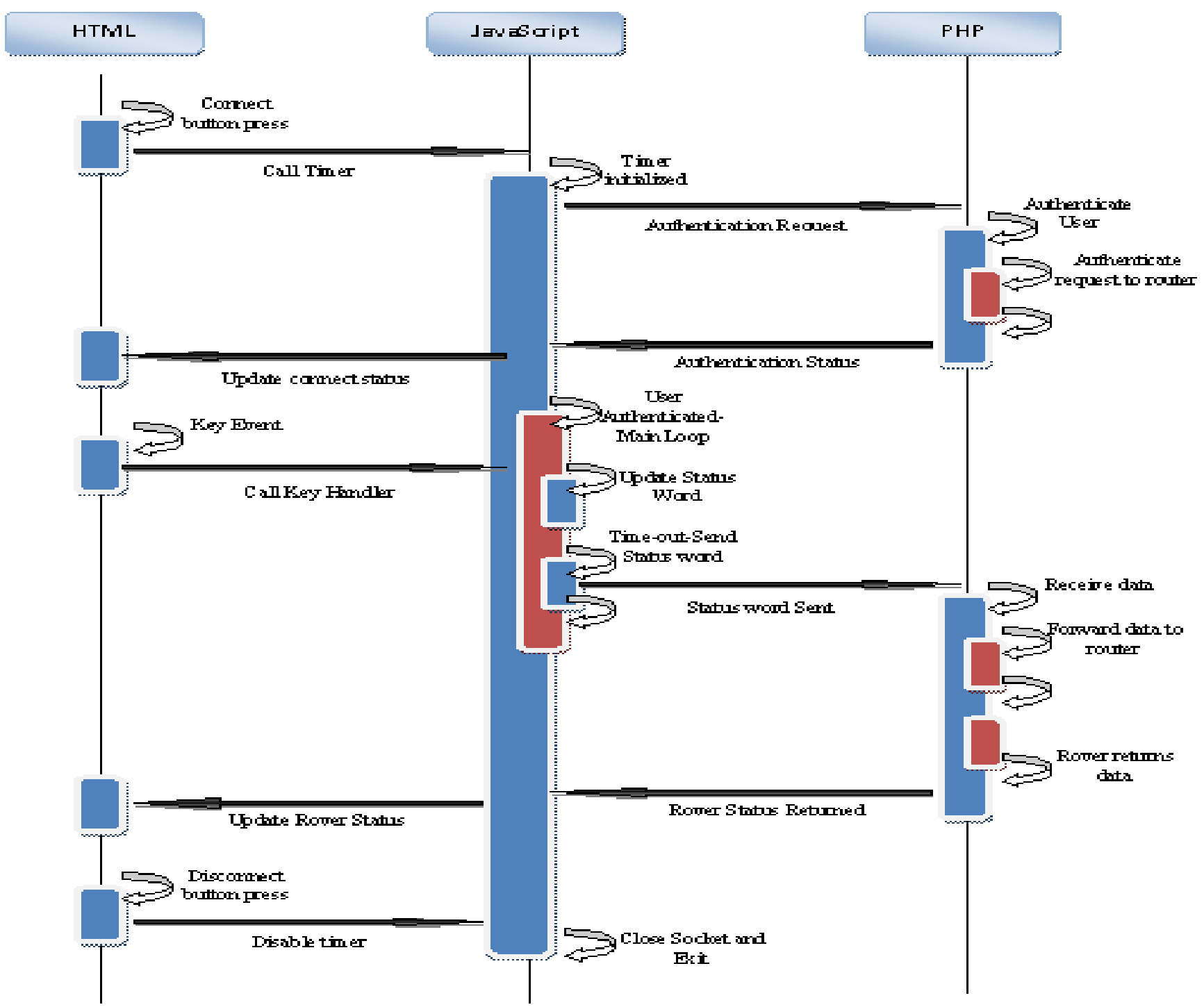

Figure 4: The Interaction Sequence diagram which shows interactions between the different components of the web client

\section{SCREENSHOTS:}

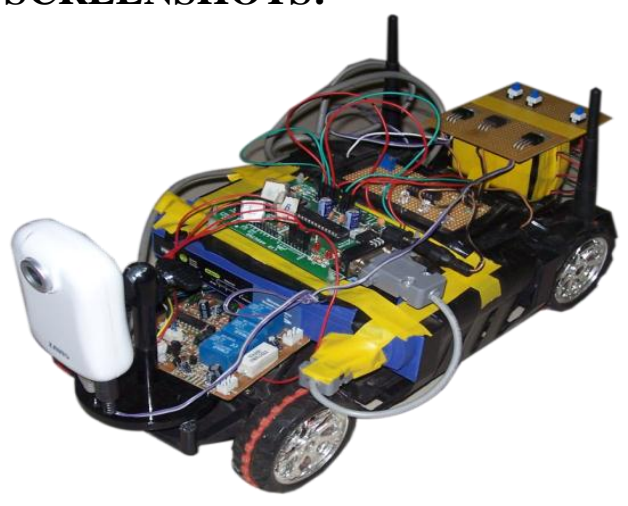

Figure 5: ROVER

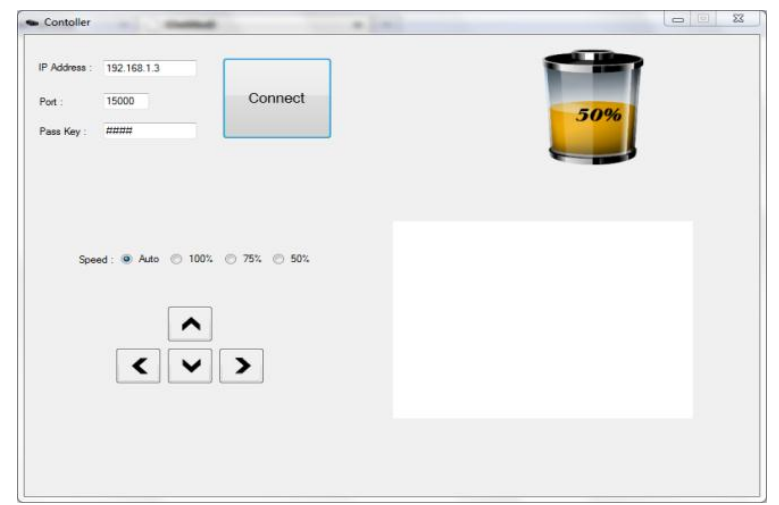

Figure 6: Desktop Client 


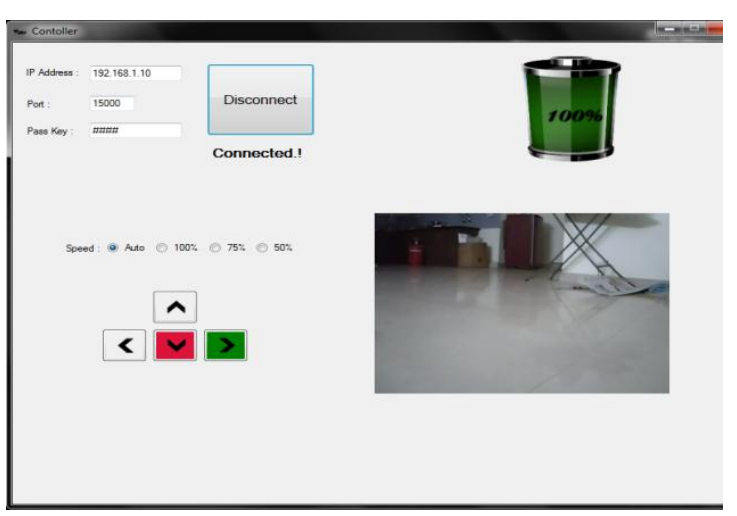

Figure 7: Desktop Client with connection established and rover moving backward right

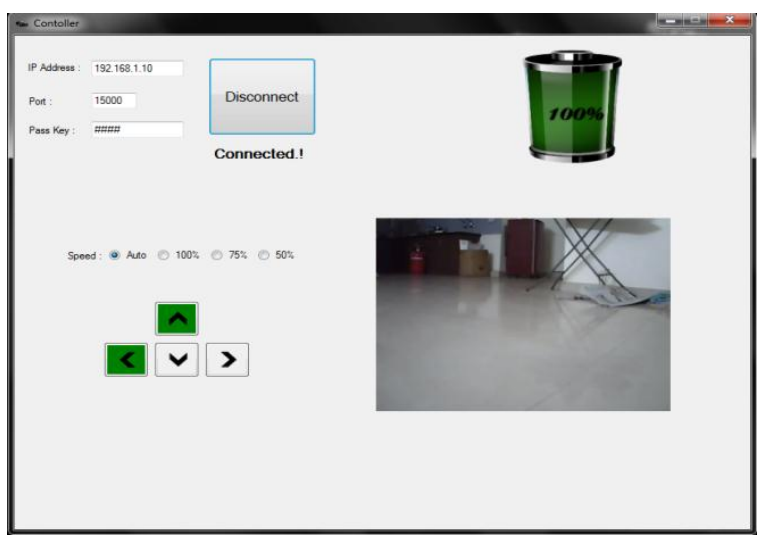

Figure 8: Desktop Client with connection established and rover moving forward left

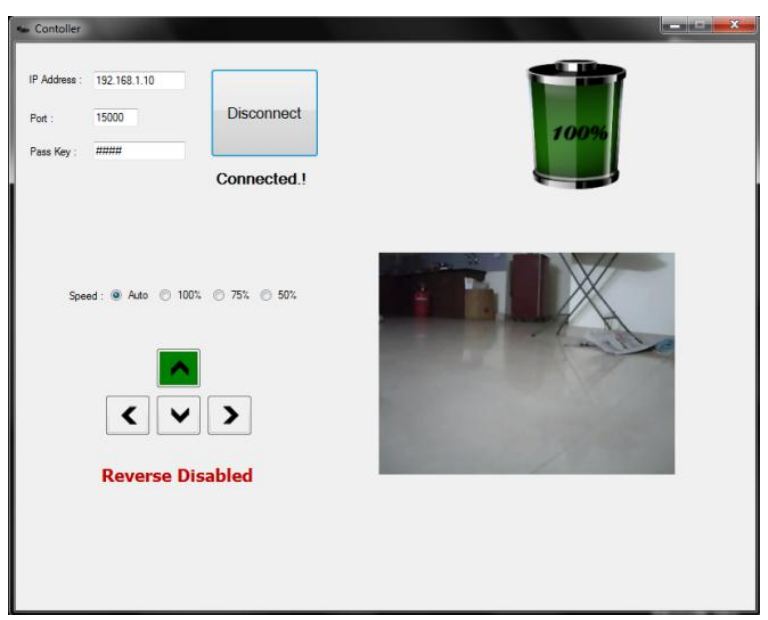

Figure 9: Desktop Client with Reverse Disable

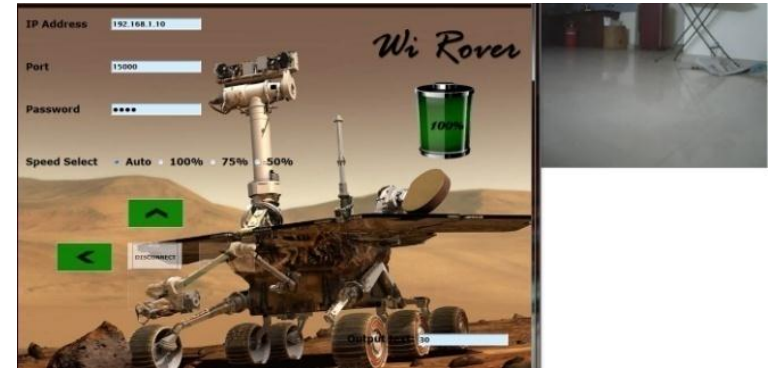

Figure 10: Web Client with connection established and rover moving forward left

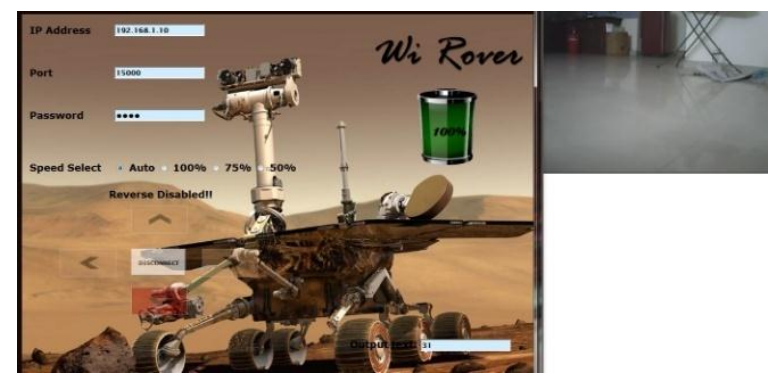

Figure 11: Web Client with Reverse Disable

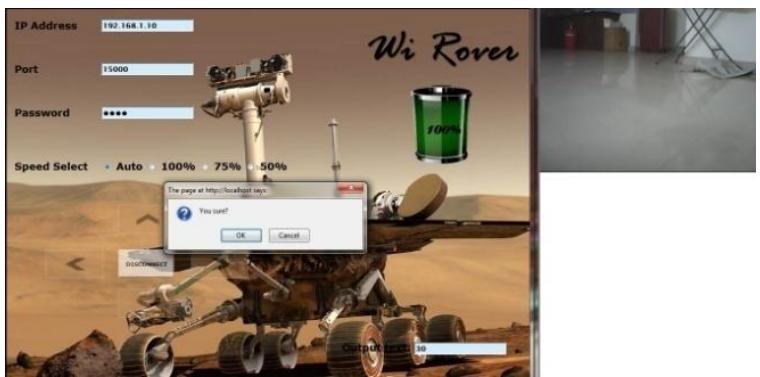

Figure 12: Web Client on Disconnection

\section{CONCLUSION AND FUTURE ENHANCEMENTS:}

A system is developed which uses wireless digital communication over the internet to control and communicate with a vehicular system. An algorithm is developed which controls the vehicle remotely and safely. It is also scalable and not so expensive. Some form of autonomous control is needed. Two-way communication is possible between the vehicle and the controller.

The future enhancements are:

- Add an interface which allows the user to set the preset path for the rover.

- Make the rover autonomous.

- To add servo motors to the rovers for accurate movement. 
- Encrypt the connection between the client and the rover.

\section{REFERENCES}

[1] Arduino Software, http://www.arduino.cc

[2] CISCO Home networking, http://www.linksys.com

[3]http://www.openwrt.org(https://forum.openwrt.org/viewtop ic.php?id=39525- Disabling powersave in compatwireless code)

[4] http://forum.openwrt.org (Add support for Linksys E1000 v1 (and brcm4716))

[5] C\# Language Pocket Reference. O' Reilly. 2002
[6] "4-D/RCS reference model architecture for unmanned ground vehicles" (PDF) http://www.isd.mel.nist.gov/documents/albus/4DRCS.pd f.

[7] Chuck Squatriglia (2008-07-01). "GM Says Driverless Cars Could Be on the Road by 2018". Wired. http://www.wired.com/autopia/2008/01/gm-saysdriverl/.Stanford University Junior car

[9] "Robot Buses Pull In to San Diego's Fastest Lane". Wired. July 24, 2007.

[10] http://www.wired.com/cars/futuretransport/magazine/1508/st_robot. 